\title{
Conjugates of Pyropheophorbide $a$ with Androgen Receptor Ligands
}

\author{
Vladimir A. Zolottsev, ${ }^{a}$ Olga V. Zazulina, ${ }^{a}$ Galina E. Morozevich, ${ }^{a}$ \\ Maria G. Zavialova, ${ }^{a}$ Alexander Y. Misharin, ${ }^{a}$ Roman A. Novikov, ${ }^{\mathrm{b}}$ \\ Vladimir P. Timofeev, ${ }^{\mathrm{b}}$ Oskar I. Koifman, ${ }^{\mathrm{c}}$ and Gelii V. Ponomareva@ \\ anstitute of Biomedical Chemistry, 119121 Moscow, Russia \\ ${ }^{\mathrm{b}}$ Engelhardt Institute of Molecular Biology, Russian Academy of Sciences, 119991 Moscow, Russia \\ ${ }^{\mathrm{c}}$ Research Institute of Macroheterocycles, Ivanovo State University of Chemistry and Technology, 153000 Ivanovo, Russia \\ @Corresponding authorE-mail: gelii@yandex.ru
}

\begin{abstract}
Two new conjugates of pyropheophorbide a with testosterone and dihydrotestosterone: $17^{3}$ [2-(17 $\beta$-hydroxy-3-oxopregn4-en-21-oylamido)ethylamido]pyropheophorbide $a$ (10) and 173 [2-(17ß-hydroxy-3-oxopregnan-21-oylamido)ethylamido]pyropheophorbide a (11) were synthesized. IC ${ }_{50}$ for conjugates 10 and 11 at 96 incubation in LNCaP and PC-3 prostate carcinoma cells were $1.4 \mu M$ and $3.3 \mu M$ for compound 10, and $4.5 \mu M$ and $6.1 \mu M$ for compound 11, respectively. Irradiation with light at wavelength of $660 \mathrm{~nm}$ increased toxicity of the conjugates.
\end{abstract}

Keywords: Conjugates, pyropheophorbide $a$, testosterone, dihydrotestosterone, prostate carcinoma cells, cytotoxicity.

\section{Конъюгаты пирофеофорбида $a$ с лиганАами андрогенового рецептора}

В. А. Золотцев, ${ }^{a}$ О. В. Зазулина, ${ }^{a}$ Г. Е. Морозевич, ${ }^{a}$ М. Г. Завьялова, ${ }^{a}$

А. Ю. Мишарин, ${ }^{a}$ Р. А. Новиков, ${ }^{\text {b }}$ В. П. Тимофреев, ${ }^{\text {b }}$ О. И. Койфрман, ${ }^{c}$

Г. В. Пономарев ${ }^{\mathrm{a}}$

\author{
${ }^{\mathrm{a}}$ Институт биомедицинской химии, 119121 Москва, Россия \\ ${ }^{\mathrm{b}}$ Институт молекулярной биологии им. В.А. Энгельгардта РАН, 119991 Москва, Россия \\ 'НИИ химии макрогетероциклических соединений, Ивановский государственньій химико-технологический \\ университет, 153000 Иваново, Россия \\ @E-mail: gelii@yandex.ru
}

\begin{abstract}
Синтезировань два новых конъюгата пирофеофорбида а с тестостероном и дигидротестостероном: $17^{3}[2-(17 \beta$-гидрокси-3-оксопрегн-4-ен-21-оиламидо)этиламидо]пирофеофорбид а (10) и 173 $[2-(17 \beta$-гидрокси-

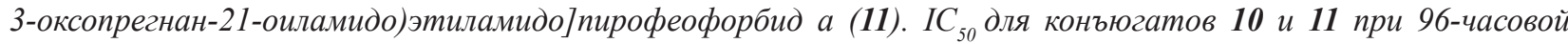
инкубаџии в клетках карииномы простаты LNCaP и PC-3 составляет 1.4 мкM и 3.3 мкM для соединения 10 и 4.5 мкМ и 6.1 мкМ для соединения 11, соответственно. Облучение светом длиной волны 660 нм приводило к многократному повымению токсичности конъюгатов.
\end{abstract}

Ключевые слова: Конъюгаты, пирофеофорбид $a$, тестостерон, дигидротестостерон, клетки карциномы простаты, цитотоксичность. 
Tetrapyrrolic macrocycles, porphyrins and chlorins, owing to their unique photochemical and photophysical properties have wide range of biomedical applications such as optical imaging, fluorescent labeling, photodynamic inactivation of microbial infections, and photodynamic therapy of solid tumors. Coupling of macrocycles with fragments of biological active molecules improves delivery and distribution of macrocycle-based compounds to a specific location within the cells, facilitates its transport through receptor or drug mediated endocytosis, and affects its biological activity. ${ }^{[1-3]}$ Synthesized earlier conjugates of macrocycles with polyamines, amino acids, peptides, peptidomimetics, antibiotics, nucleotides, carbohydrates, bile acids, lipids, steroids, etc., revealed prospective implications in biomedical studies and photodynamic therapy. ${ }^{[4-19]}$

In this study we have synthesized conjugates of pyropheophorbide $a$ with androgen receptor ligands testosterone and dihydrotestosterone. Androgen receptor is known to be an important drug target for treatment of prostate cancer. Modern trends in preparation and application of various steroid conjugates targeting androgen receptor have been reviewed. ${ }^{[20,}$ and the ref. therein] Until now conjugates of testosterone and dihydrotestosterone with tetrapyrrolic macrocycles have not been reported. Synthesis of new conjugates $\mathbf{1 0}$ and $\mathbf{1 1}$ is presented in the Scheme 1.

Testosterone $\mathbf{1}$ and dihydrotestosterone $\mathbf{4}$ were transformed to steroid blocks $\mathbf{2}$ and $\mathbf{5}$ by three steps including consecutive protection of carbonyl functions with formation of 1,3-dioxolanes, oxidation of 17 $\beta$-hydroxyl groups, and Reformatsky reaction of obtained 17-ketones with $\mathrm{Zn}$ and ethyl bromoacetate. ${ }^{[21,22]}$ The aforementioned reaction is known to pass stereoselectively and give appropriate $17 \beta-\mathrm{OH}$ isomer. Removal of ethylene ketal and ethyl ester protective groups in compounds $\mathbf{2}$ and $\mathbf{5}$ led to 21-carboxylic acids 3 and $\mathbf{6}$ in $49 \%$ and $58 \%$ overall yields (based on compounds 1 and $\mathbf{4}$, respectively). Compounds 3 and $\mathbf{6}$ were transformed to related $N$-hydroxysuccinimide esters $\mathbf{3 a}$ and $\mathbf{6 a}$ by treatment with $N$-hydroxysuccinimide in the presence of $N, N^{\prime}$-dicyclohexylcarbodiimide (DCC). HRMS, ${ }^{1} \mathrm{H}$ NMR, ${ }^{13} \mathrm{C}$ NMR data for compounds $\mathbf{2}, \mathbf{3}, \mathbf{3 a}, \mathbf{5}$, $\mathbf{6}$ and $\mathbf{6} \mathbf{a}$ are given in Supplementary section.

Pyropheophorbide $a$ derivative comprising primary amino group (compound $\mathbf{8}^{[23]}$ ) was prepared from pyropheophorbide $a 7$ through formation of pentafluorophenyl ester 7a, followed by its treatment with excess of ethylene diamine. Compound $\mathbf{9}^{\text {[24] }}$ comprising Boc-protected amino group was prepared from pentafluorophenyl ester 7a by same reaction with mono-Boc ethylene diamine. ${ }^{[25]}$

Condensation of $\mathrm{N}$-hydroxysuccinimide esters of steroid acids $\mathbf{3 a}$ and $\mathbf{6 a}$ with $17^{3}$ [(2-aminoethyl)amido] pyropheophorbide $a(\mathbf{8})$ led to the target conjugates $\mathbf{1 0}$ and 11, ${ }^{[26,27]}$ respectively. These conjugates were isolated as individual compounds. Their structures were completely characterized by HRMS, ${ }^{1} \mathrm{H}$ NMR, ${ }^{13} \mathrm{C}$ NMR and electron absorption spectra.

Absorption spectra of conjugates $\mathbf{9 ,} \mathbf{1 0}$ and $\mathbf{1 1}$ in $\mathrm{CH}_{2} \mathrm{Cl}_{2}$ were very close to those for pyropheophorbide $a$ 7 and $17^{3}[(2$-aminoethyl)amido]pyropheophorbide $a$ (8). ${ }^{1} \mathrm{H}$ NMR spectra of conjugates $\mathbf{1 0}$ and $\mathbf{1 1}$ displayed strong high field shifts for H-18' and H-19' methyl protons in comparison with those in spectra of non conjugated steroids (s, $0.53 \mathrm{ppm}$ and s, $0.91 \mathrm{ppm}$ for compound $\mathbf{1 0}$ instead of s, $0.95 \mathrm{ppm}$ and s, $1.19 \mathrm{ppm}$ for compound 3 ; s, $0.56 \mathrm{ppm}$ and $\mathrm{s}$, $0.78 \mathrm{ppm}$ for compound 11 instead of s, 0.92 and s, $1.01 \mathrm{ppm}$ for compound 6). The H-4' resonance for compound $\mathbf{1 0}$ (s,
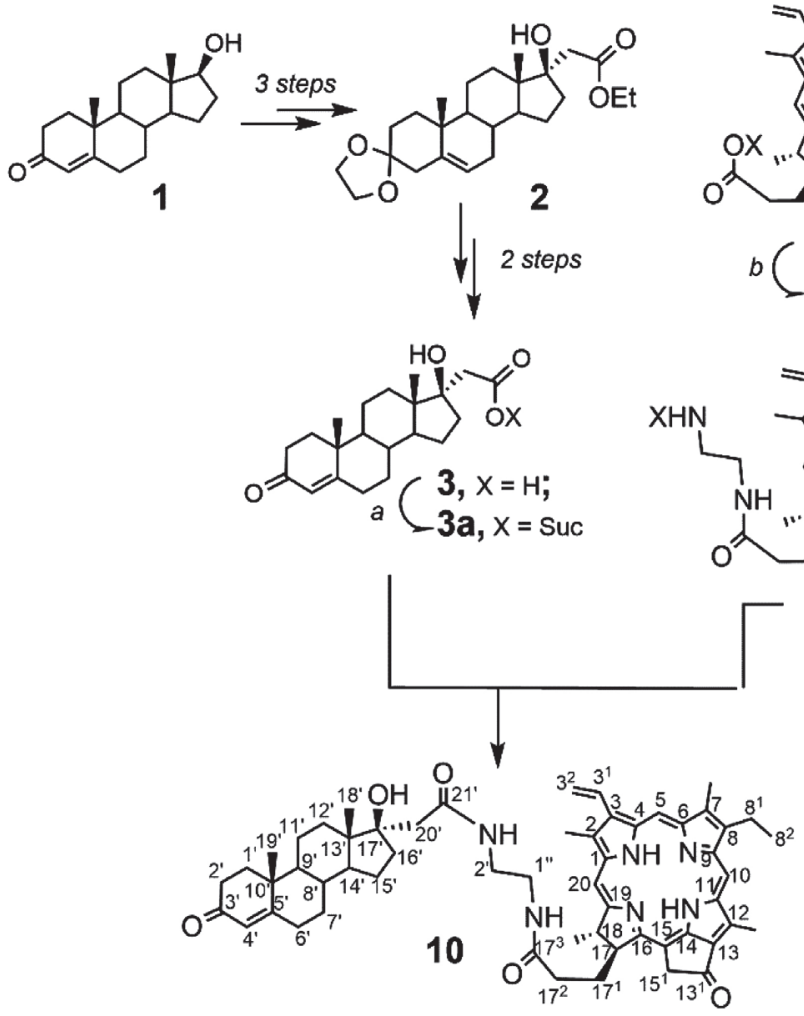

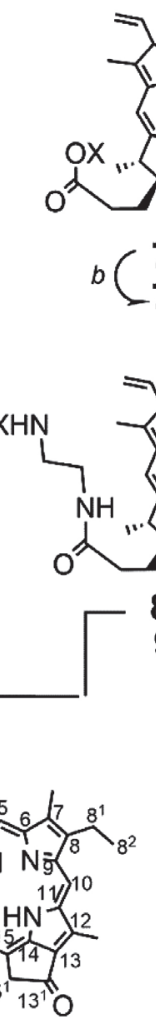

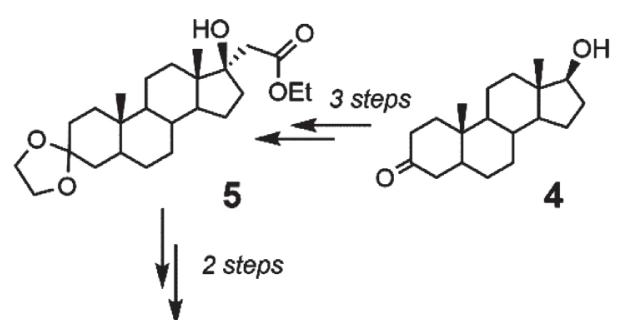
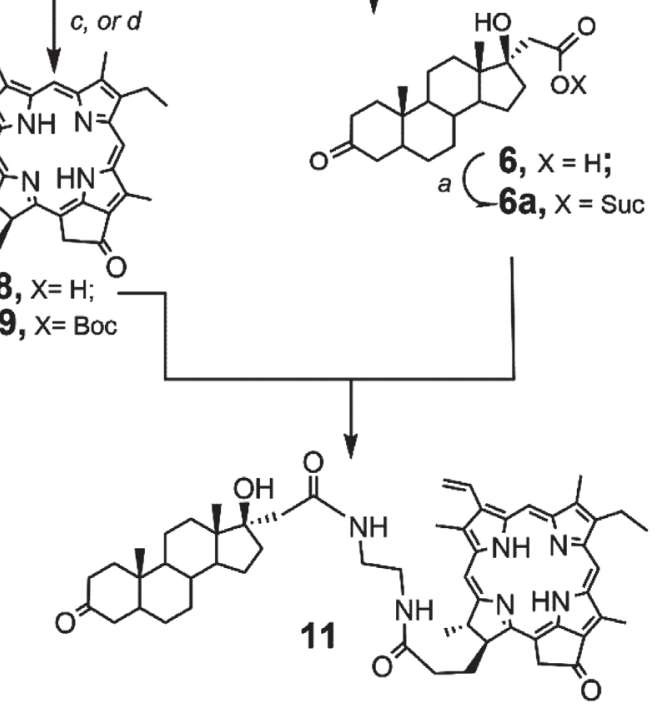

Scheme 1. (a) N-OSu, DCC/CH $\mathrm{Cl}_{2}$; (b) $\mathrm{CF}_{3} \mathrm{COOC}_{6} \mathrm{~F}_{5} / \mathrm{CH}_{2} \mathrm{Cl}_{2}$; (c) $\mathrm{H}_{2} \mathrm{~N}\left(\mathrm{CH}_{2}\right)_{2} \mathrm{NH}_{2} / \mathrm{CH}_{2} \mathrm{Cl}_{2}$; (d) $\mathrm{BocNH}\left(\mathrm{CH}_{2}\right)_{2} \mathrm{NH}_{2} / \mathrm{CH}_{2} \mathrm{Cl}_{2}$. 
$5.46 \mathrm{ppm}$ ) was also shifted in high field compared with those for compound $3(\mathrm{~s}, 5.73 \mathrm{ppm})$. These spectral peculiarities apparently were caused by influence of macrocycle on steroid moiety; close effects were reported earlier for conjugates of pyropheophorbide $a$ with cholesterol. ${ }^{[28]}$ Resonance of tertbutyl protons in conjugate 9 (s, $1.21 \mathrm{ppm}$ ) was also shifted in high field compared to those usually observed for Bocamides (s, 1.4 ppm).

Speculating that steroid fragments may affect affinity conjugates $\mathbf{1 0}$ and $\mathbf{1 1}$ to prostate carcinoma cells, we investigated viability of androgen-sensitive $\mathrm{LNCaP}$ and androgen-insensitive PC-3 cells in the presence of these conjugates and $17^{3}[(2$ "-tert-butyloxycarbonylamidoethyl)amido]pyropheophorbide $a$ (9) (as reference compound). Two experiments were carried out: in the Experiment 1 we have measured LNCaP and PC-3 cells viability at $96 \mathrm{~h}$ incubation with compounds 9, 10 and 11; in the Experiment 2 we have compared dark toxicity and photo toxicity of conjugates in the same cells at short time incubation (labeling - $18 \mathrm{~h}$; irradiation - $10 \mathrm{~min}$; incubation without compounds 24 h). Cell viability was measured with MTT method. ${ }^{29]}$ The protocol used is given in supplementary section. Student's $t$-test was used to estimate average values for all cases. All Student's $t$-tests were calculated by an online calculator (http://www.graphpad.com/quickcalcs/ttest1. $c f m$ ), confidence interval for each case did not exceed $6 \%$ of the mean.

The results demonstrated that coupling of pyropheophorbide $a$ with testosterone and dihydrotestosterone led to conjugates toxic in LNCaP and PC-3 cells. Figures $1 \mathrm{a}$ and $1 \mathrm{~b}$ (see Supplementary section) showed that conjugates $\mathbf{1 0}$ and 11 were highly toxic in both prostate carcinoma cells at $96 \mathrm{~h}$ incubation; conjugate $\mathbf{1 0}$ being significantly more potent cytotoxic agent than conjugate 11, steroid-free conjugate 9 exhibited rather low effect on cells viability.

Figures $1 \mathrm{c}$ and $1 \mathrm{~d}$ showed that both conjugates $\mathbf{1 0}$ and 11 decreased LNCaP and PC-3 cells viability at short time incubation (dark toxicity), though less potently than at $96 \mathrm{~h}$ incubation; conjugate 9 at short time incubation stimulated proliferation of LNCaP, rather than PC-3 cells. Irradiation (LED AFS "Spectrum", Laser medical centrum Ltd, Moscow, Russia; wavelength of $660 \mathrm{~nm}, 10 \mathrm{~min}$ ) potently increased toxicity of conjugates in all cases. However, at short time incubation (either with irradiation, or without irradiation) cells viability remained rather high $(\approx 20 \%$ for PC-3 cells, $\approx 40 \%$ for $\mathrm{LNCaP}$ cells) even at $50 \mu \mathrm{M}$ and $100 \mu \mathrm{M}$ of conjugates $\mathbf{1 0}$ and $\mathbf{1 1}$. $\mathrm{IC}_{50}$ for conjugates $\mathbf{1 0}$ and 11 at 96 h incubation in LNCaP and PC-3 prostate carcinoma cells were $1.4 \mu \mathrm{M}$ and $3.3 \mu \mathrm{M}$ for compound $\mathbf{1 0}$, and 4.5 $\mu \mathrm{M}$ and $6.1 \mu \mathrm{M}$ for compound $\mathbf{1 1}$, respectively (Table 1, Supplementary section).

In cocnclusion, conjugates of pyropheophorbide $a$ with androgen receptor ligands - testosterone and dihydrotestosterone - were synthesized. These conjugates were found to exhibit potent dark and photo toxicity in prostate carcinoma cells. We speculate that further investigation of uptake, distribution, subcellular localization, and possible participation in signaling and regulatory pathways of these compounds and related steroid conjugates may be helpful for development of new photo sensitizers possessing high specificity and activity.
Acknowledgments. Authors acknowledge Mr. Mikhail Muraviev, the head of "Laser medical centrum Ltd", kindly providing LED AFS "Spectrum" for photo toxicity experiments. This work was supported by Russian Foundation for Basic Research (project No 15-04-02426), Russian Science Foundation (project No. 14-23-00204) and Programs for Basic Research of Russian State Academy of Sciences for 2013-2020 and "Molecular and cell biology" of Presidium of Russian Academy of Science.

\section{References and Notes}

1. Sharman W.M., van Lier J.E., Allen C. M. Adv. Drug Deliv. Rev. 2004, 56, 53 .

2. Chari R.V., Acc. Chem. Res. 2008, 41, 98

3. Schneider R., Tirand L., Frochot C., Vanderesse R., Thomas N., Gravier J., Guillemin F., Barberi-Heyob M. Anticancer Agents Med. Chem. 2006, 6, 469.

4. Sibrian-Vazquez M., Jensen T.J., Fronczek F.R., Hammer R.P., Vicente M.G.H. Bioconjug. Chem. 2005, 16, 852.

5. Hargus J.A., Fronczek F.R., Vicente M.G.H., Smith K.M. Photochem. Photobiol. 2007, 83, 1006.

6. Sibrian-Vazquez M., Jensen T.J., Vicente M.G.H. Org. Biomol. Chem. 2010, 8, 1160.

7. Dmitriev R.I., Ropiak H.M., Ponomarev G.V., Yashunsky D.V., Papkovsky D.B. Bioconjug. Chem. 2011, 22, 2507.

8. Jinadasa W.R.G., Hu X., Vicente M.G.H., Smith K.M. J. Med. Chem. 2011, 54, 7464.

9. Jinadasa W.R.G., Zhou Z., Vicente M.G.H., Smith K.M. Org. Biomol. Chem. 2016, 14, 1049.

10. Zheng X., Morgan J., Pandey S.K., Chen Y., Tracy E., Baumann H., Missert J.R., Batt C., Jackson J., Bellnier D.A., Henderson B.W., Pandey R.K. J. Med. Chem. 2009, 52, 4306.

11. Nikolaeva I.A., Misharin A.Y., Ponomarev G.V., Timofeev V.P., Tkachev Y.V. Bioorg. Med. Chem. Lett. 2010, 20, 2872.

12. Nikolaeva I.A., Morozova J.V., Zavialova M.G., Novikov R.A., Tkachev Y.V., Timofeev V.P., Misharin A.Y., Ponomarev G.V. Macroheterocycles 2010, 3, 150.

13. Battogtokh G., Liu H-B., Bae S-M., Chaturvedi P.K., Kim Y.W., Kim I-W., Ahn W.S. J. Photochem. Photobiol., B 2012, 110, 50.

14. Hahn F., Schmitz K., Balaban T.S., Brase S., Schepers U. ChemMedChem. 2008, 3, 1185.

15. Kascakova S., Hofland L.J., De Bruijn H.S., Ye Y., Achilefu S., van der Wansem K., van der Ploeg-van den Heuvel A., van Koetsveld P.M., Brugts M.P., van der Lelij A-J., Sterenborg H.J. C.M., ten Hagen T.L.M., Robinson D.J., van Hagen M.P. PLoS One 2014, 9(8), e104448.

16. Khan E.H., Ali H., Tian H., Rousseau J., Tessier G., Shafiullaha, van Lier J.E. Bioorg. Med. Chem. Lett. 2003, 13, 1287.

17. Koivukorpi J., Sievänen E., Kolehmainen E., Král V. Molecules 2007, 12, 13 .

18. Mammana A., Asakawa T., Bitsch-Jensen K-B., Wolfe A., Chaturantabut S., Otani Y., Li X., Li Z., Nakanishi K., Balaz M., Ellestad G. A., Berova N. Bioorg. Med. Chem. 2008, 16, 6544.

19. Zhylitskaya H.A., Zhabinskii V.N., Litvinovskaya R.P., Lettieri R., Monti D., Venanzi M., Khripach V.A., Drašar P. Steroids 2012, 77, 1169.

20. Levine P.M., Garabedian M.J., Kirshenbaum K. J. Med. Chem. 2014, 57, 8224 .

21. Bittler D., Laurent H., Rach P.,Topert, M. US Patent 5010071 , Apr. 23, 1991

22. Oliveto E.P. In: Organic Reactions in Steroid Chemistry, Vol. II (Fried J., Edwards J.A., Eds.). Van Nostrand Reinhold Co.; 1972. p. 139. 
23. $17^{3}[(2 "$-Aminoethyl)amido]pyropheophorbide a (8). The mixture of pentafluorophenylpyropheophorbide $a 7 \mathbf{7}(202 \mathrm{mg}$, $0.29 \mathrm{mmol})$, ethylene diamine $(580 \mu \mathrm{L}, 520 \mathrm{mg}, 8.65 \mathrm{mmol})$ and abs. $\mathrm{CH}_{2} \mathrm{Cl}_{2}(10 \mathrm{~mL})$ was stirred for $2 \mathrm{~h}$, then the mixture was poured into $0.1 \mathrm{M} \mathrm{CH}_{3} \mathrm{COONa}$ buffer $(\mathrm{pH} 5.20 \mathrm{~mL})$, extracted with $\mathrm{CH}_{2} \mathrm{Cl}_{2}(2 \times 20 \mathrm{~mL})$, the combined extract was washed with brine $(20 \mathrm{~mL})$, dried over $\mathrm{Na}_{2} \mathrm{SO}_{4}$, and evaporated. Then the residue was dissolved in THF $(30 \mathrm{~mL})$, the solution was dried over granulated $\mathrm{KOH}$, followed by evaporation to dryness. The obtained black powder (139 mg, $0.24 \mathrm{mmol}, 83 \%$ ) was used without further purification; the analytical sample was purified by TLC in $\mathrm{CHCl}_{3}: \mathrm{MeOH}: \mathrm{NH}_{4} \mathrm{OH}$ (90:9:1) mixture. HRMS, calculated for $\left[\mathrm{C}_{35} \mathrm{H}_{41} \mathrm{~N}_{6} \mathrm{O}_{2}\right]^{+}: 577.3291$, found: $577.3292 .{ }^{1} \mathrm{H}$ NMR $\delta$ ppm: $-1.70,0.33$ (each $1 \mathrm{H}$, br.s, $\mathrm{N}-\mathrm{H}) ; 1.62(3 \mathrm{H}, \mathrm{t}$, $\left.J=7.6 \mathrm{~Hz}, 8^{2}-\mathrm{H}\right) ; 1.75\left(3 \mathrm{H}, \mathrm{d}, J=7.3 \mathrm{~Hz}, 18-\mathrm{CH}_{3}\right) ; 3.18,3.37$, 3.41 (each $\left.3 \mathrm{H}, \mathrm{s}, 2-, 7-, 12-\mathrm{CH}_{3}\right) ; 4.23,4.45$ (each $1 \mathrm{H}, \mathrm{m}$, $17^{1}-\mathrm{H}$ and $8^{1}-\mathrm{H}$ ); 4.98, 5.19 (each $1 \mathrm{H}, \mathrm{d}, J=19.7 \mathrm{~Hz}, 17^{2}-\mathrm{H}$ ); $6.13\left(1 \mathrm{H}, \mathrm{dd}, J=11.5 \mathrm{~Hz}\right.$ and $\left.J=1.4 \mathrm{~Hz}, 3^{2}-\mathrm{H}, c i s\right) ; 6.24(1 \mathrm{H}$, dd, $J=17.9 \mathrm{~Hz}$ and $J=1.4 \mathrm{~Hz}, 3^{2}-\mathrm{H}$, trans $) ; 7.95(1 \mathrm{H}$, dd, $J=11.5 \mathrm{~Hz}$ and $J=17.9 \mathrm{~Hz}, 3^{1}-\mathrm{H}$ ); 8.50, 9.24, 9.30 (each $1 \mathrm{H}, \mathrm{s}$, 5-, $10-, 20-\mathrm{H}) ;{ }^{13} \mathrm{C}$ NMR $\delta$ ppm: $11.18 ; 11.81 ; 12.05 ; 17.36$; 19.37 ; $23.01 ; 28.30 ; 30.17 ; 30.88 ; 32.80 ; 40.92 ; 41.65 ; 48.01$; $49.97 ; 51.70 ; 92.92 ; 97.08 ; 103.88 ; 106.03 ; 122.65 ; 128.10$; $129.18 ; 131.50 ; 135.78 ; 135.96 ; 136.13 ; 137.68 ; 144.92$; $148.86 ; 150.65 ; 155.11 ; 160.37 ; 171.68 ; 172.36 ; 196.14$. UV-Vis $\left(\mathrm{CH}_{2} \mathrm{Cl}_{2}\right) \lambda_{\max } \mathrm{nm}(\varepsilon): 413$ (85,000); $507(8,900) ; 538$ $(8,000) ; 609(7,000) ; 665(35,200)$.

24. $17^{3}[(2$ '”-tert-Butyloxycarbonylamidoethyl) amido]pyropheophorbide a (9). Compound 9 was synthesized from pentafluorophenylpyropheophorbide $a$ 7a $(88 \mathrm{mg}, 0.13 \mathrm{mmol})$ and mono-Boc-ethylene diamine $(42 \mathrm{mg}, 0.26 \mathrm{mmol}$ ) according the procedure described in ref. ${ }^{[29]}$ and isolated by silica gel flash chromatography in $\mathrm{CHCl}_{3}: \mathrm{MeOH}: \mathrm{NH}_{4} \mathrm{OH}$ (90:9:1) mixture. After evaporation compound 9 (43 mg, $0.06 \mathrm{mmol}$, $43 \%$ ) was obtained as black powder. HRMS, calculated for $\left[\mathrm{C}_{40} \mathrm{H}_{49} \mathrm{~N}_{6} \mathrm{O}_{4}\right]^{+}: 677.3815$, found: 677.3818 . ${ }^{1} \mathrm{H}$ NMR $\delta \mathrm{ppm}$ : $-1.74,0.36$ (each $1 \mathrm{H}$, br.s, $\mathrm{N}-\mathrm{H}) ; 1.21(9 \mathrm{H}, \mathrm{s}, t-\mathrm{Bu}) ; 1.59(3 \mathrm{H}$, t, $\left.J=7.6 \mathrm{~Hz}, 8^{3}-\mathrm{H}\right) ; 1.76\left(3 \mathrm{H}, \mathrm{d}, J=7.3 \mathrm{~Hz}, 18-\mathrm{CH}_{3}\right) ; 3.17,3.27$, 3.37 (each $\left.3 \mathrm{H}, \mathrm{s}, 2-, 7-, 12-\mathrm{CH}_{3}\right) ; 4.25,4.47$ (each 1H, m, $17^{1}-\mathrm{H}$ and $8^{1}-\mathrm{H}$ ); 5.01, 5.21 (each $1 \mathrm{H}, \mathrm{d}, J=19.7 \mathrm{~Hz}, 17^{2}-\mathrm{H}$ ); $6.12\left(1 \mathrm{H}, \mathrm{dd}, J=11.5 \mathrm{~Hz}\right.$ and $\left.J=1.4 \mathrm{~Hz}, 3^{2}-\mathrm{H}, c i s\right) ; 6.23(1 \mathrm{H}$, dd, $J=17.9 \mathrm{~Hz}$ and $J=1.4 \mathrm{~Hz}, 3^{2}-\mathrm{H}$, trans); 7.92 (each $1 \mathrm{H}$, dd, $J=11.5 \mathrm{~Hz}$ and $J=17.9 \mathrm{~Hz}, 3^{1}-\mathrm{H}$ ); 8.51, 9.12, 9.28 (each $1 \mathrm{H}, \mathrm{s}$, 5-, $10-, 20-\mathrm{H}) .{ }^{13} \mathrm{C}$ NMR $\delta$ ppm: $11.25 ; 11.76 ; 12.14 ; 17.41$; $19.04 ; 23.15 ; 28.24 ; 28.46 ; 30.44 ; 33.02 ; 40.36 ; 40.63 ; 48.09$; $50.08 ; 51.85 ; 79.56 ; 93.07 ; 97.14 ; 103.89 ; 106.08 ; 122.55$; $128.06 ; 129.25 ; 130.28 ; 131.63 ; 135.86 ; 135.99 ; 136.23$; $137.89 ; 141.61 ; 145.01 ; 148.95 ; 150.69 ; 155.19 ; 160.50$; $171.80 ; 173.01 ; 196.26$. UV-Vis $\left(\mathrm{CH}_{2} \mathrm{Cl}_{2}\right) \lambda_{\max } \mathrm{nm}(\varepsilon): 413$ (85,000); 507 (8,900); 538 (8,000); 609 (7,000); $665(35,200)$.

25. Krapcho A.P., Kuell C.S. Synth. Commun. 1990, 20, 2559.

26. $17^{3}[2$ '”-(17' $\beta$-Hydroxy-3'-oxopregn-4'-en-21'-oylamidoethyl) amido]pyropheophorbide a (10): The mixture of compounds 3a (30 mg, $69 \mu \mathrm{mol}), 8$ (33 mg, $57 \mu \mathrm{mol})$, dry Py (3 mL), and dry THF $(5 \mathrm{~mL})$ was stirred at $\mathrm{r}$. t. for $16 \mathrm{~h}$, then evaporated to dryness with toluene, and the residue was applied on the top a silica gel column. The column initially was washed with $\mathrm{CHCl}_{3}:\left(\mathrm{CH}_{3}\right)_{2} \mathrm{CO}: \mathrm{AcOH}(75: 24: 1)$ to remove byproducts, then washed with $5 \mathrm{~mL} \mathrm{CHCl}_{3}$, and finally the target product was eluted with $\mathrm{CHCl}_{3}: \mathrm{MeOH}_{3}: 7 \mathrm{M} \mathrm{NH}_{3}$ solution in $\mathrm{MeOH}$ (93:5:2, by vol). After evaporation the compound $\mathbf{1 0}$ (38 mg, $42 \mu \mathrm{mol}$, $73 \%$ ) was obtained as black powder. HRMS, calculated for $\left[\mathrm{C}_{56} \mathrm{H}_{69} \mathrm{~N}_{6} \mathrm{O}_{5}\right]^{+}: 905.5329$, found: 905.5327. ${ }^{1} \mathrm{H}$ NMR $\delta$ ppm: $-1.86(1 \mathrm{H}$, br.s, $\mathrm{N}-\mathrm{H}) ; 0.53,0.91$ (each $3 \mathrm{H}, \mathrm{s}, \mathrm{H}-18^{\prime}$ and $\mathrm{H}-19^{\prime}$ in steroid moiety); $1.61\left(3 \mathrm{H}, \mathrm{t}, J=7.6 \mathrm{~Hz}, 8^{2}-\mathrm{H}\right.$ in pyropheophorbide moiety), $1.74\left(3 \mathrm{H}, \mathrm{d}, J=7.3 \mathrm{~Hz}, 18-\mathrm{CH}_{3}\right.$ in pyropheophorbide moiety), 3.19, 3.36, 3.39 (each 3H, s, 2-, $7-, 12-\mathrm{CH}_{3}$ in pyropheophorbide moiety), 4.23, 4.46 (each $1 \mathrm{H}$, $\mathrm{m}, 17^{1}-\mathrm{H}$ and $8^{1}-\mathrm{H}$ in pyropheophorbide moiety), 4.98, 5.17 (each $1 \mathrm{H}, \mathrm{d}, J=19.7 \mathrm{~Hz}, 17^{2}-\mathrm{H}$ in pyropheophorbide moiety), $5.46(1 \mathrm{H}, \mathrm{s}, \mathrm{H}-4$ ' in steroid moiety), $6.15(1 \mathrm{H}, \mathrm{dd}, J=11.5 \mathrm{~Hz}$ and $J=1.4 \mathrm{~Hz}, 3^{2}-\mathrm{H}$, cis in pyropheophorbide moiety), 6.17 $(1 \mathrm{H}$, br. t, $J=5.2 \mathrm{~Hz}, \mathrm{NH}-\mathrm{CO}) ; 6.25(1 \mathrm{H}, \mathrm{dd}, J=17.9 \mathrm{~Hz}$ and $J=1.4 \mathrm{~Hz}, 3^{2}-\mathrm{H}$, trans in pyropheophorbide moiety), 6.71 $(1 \mathrm{H}$, br.t, $J=5.2 \mathrm{~Hz}, \mathrm{NH}-\mathrm{CO}) ; 7.90(1 \mathrm{H}, \mathrm{dd}, J=11.5 \mathrm{~Hz}$ and $J=17.9 \mathrm{~Hz}, 3^{1}-\mathrm{H}$ in pyropheophorbide moiety), 8.58, 9.30, 9.35 (each 1H, s, 5-, 10-, 20-H in pyropheophorbide moiety). ${ }^{13} \mathrm{C}$ NMR $\delta$ ppm: $11.29 ; 12.00 ; 12.15 ; 13.60 ; 17.16 ; 17.32$; $19.51 ; 20.36 ; 20.51 ; 23.17 ; 23.31 ; 30.35,31.32 ; 32.59 ; 32.78$; $33.83 ; 35.46 ; 36.00 ; 38.42 ; 39.59 ; 42.43 ; 45.97 ; 48.10 ; 49.62$; $50.10 ; 51.92 ; 53.33 ; 81.77 ; 93.88 ; 97.10 ; 103.93 ; 106.51$; $123.09 ; 123.67 ; 128.30 ; 129.04 ; 130.53 ; 132.19 ; 135.81$; $136.35 ; 136.50 ; 137.83 ; 141.97 ; 144.94 ; 146.94 ; 149.36$, $153.65 ; 155.65,161.35 ; 171.17 ; 172.46 ; 173.59 ; 173.84$; $174.03 ; 196.12 ; 199.41$. UV-Vis $\left(\mathrm{CH}_{2} \mathrm{Cl}_{2}\right) \lambda_{\max } \mathrm{nm}(\varepsilon): 413$ $(86,400) ; 507$ (8,700); 538 (7,800); 609 (6,900); $667(36,000)$.

27. $17^{3}[2$ '-(17' $\beta$-Hydroxy-3'-oxopregnan-21'-oylamidoethyl) amido]pyropheophorbide a (11). The synthesis of compound 11 was carried out from compounds $6 \mathbf{6}(26 \mathrm{mg}, 60 \mu \mathrm{mol})$ and $8(30 \mathrm{mg}, 52 \mu \mathrm{mol})$ using the procedure described in ref. ${ }^{[25]}$ Compound 11 (33 mg, $37 \mu \mathrm{mol}, 69 \%$ ) was obtained as black powder. HRMS, calculated for $\left[\mathrm{C}_{56} \mathrm{H}_{71} \mathrm{~N}_{6} \mathrm{O}_{5}\right]^{+}$: 907.5486, found: $907.5490 .{ }^{1} \mathrm{H}$ NMR $\delta$ ppm: $-1.67,(1 \mathrm{H}$, br.s, N-H); $0.56,0.78$ (each $3 \mathrm{H}, \mathrm{s}, \mathrm{H}-18^{\prime}$ and $\mathrm{H}-19^{\prime}$ ' in steroid moiety); $1.65\left(3 \mathrm{H}, \mathrm{t}, J=7.6 \mathrm{~Hz}, 8^{2}-\mathrm{H}\right.$ in pyropheophorbide moiety); 1.77 (3H, d, $J=7.3 \mathrm{~Hz}, 18-\mathrm{CH}_{3}$ in pyropheophorbide moiety); 3.21, $3.37,3.45$ (each $3 \mathrm{H}, \mathrm{s}, 2-, 7-, 12-\mathrm{CH}_{3}$ in pyropheophorbide moiety); 4.27, 4.46 (each $1 \mathrm{H}, \quad \mathrm{m}, 17^{1}-\mathrm{H}, 8^{1}-\mathrm{H}$ in pyropheophorbide moiety); 5.02, 5.21 (each $1 \mathrm{H}, \mathrm{d}, J=19.7$ $17^{2}-\mathrm{H}$ in pyropheophorbide moiety); $5.86(1 \mathrm{H}$, br.t, $J=5.2 \mathrm{~Hz}$, $\mathrm{NH}-\mathrm{CO}) ; 6.14\left(1 \mathrm{H}, \mathrm{dd}, J=11.5 \mathrm{~Hz}\right.$ and $J=1.4 \mathrm{~Hz}, 3^{2}-\mathrm{H}$, cis in pyropheophorbide moiety), $6.20(1 \mathrm{H}$, dd, $J=17.9 \mathrm{~Hz}$ and $J=1.4 \mathrm{~Hz}, 3^{2}-\mathrm{H}$, trans in pyropheophorbide moiety), 6.58 $(1 \mathrm{H}$, br. t, $J=5.2 \mathrm{~Hz}, \mathrm{NH}-\mathrm{CO}) ; 7.93(1 \mathrm{H}, \mathrm{dd}, J=11.5 \mathrm{~Hz}$ and $J=17.9 \mathrm{~Hz}, 3^{1}-\mathrm{H}$ in pyropheophorbide moiety), 8.52, 9.30, 9.33 (each $1 \mathrm{H}, \mathrm{s}, 5-, 10-, 20-\mathrm{H}$ in pyropheophorbide moiety). ${ }^{13} \mathrm{C}$ NMR $\delta$ ppm: $11.39 ; 12.03 ; 13.62 ; 14.02 ; 17.34 ; 19.31$; $20.61 ; 23.05 ; 23.36 ; 28.42 ; 28.74 ; 29.69 ; 31.46 ; 31.87 ; 32.98$; $33.90 ; 35.25 ; 35.66 ; 36.16 ; 38.48 ; 39.72 ; 42.49 ; 46.00 ; 46.63$; $48.04 ; 49.95 ; 50.03 ; 51.72 ; 53.67 ; 81.85 ; 93.11 ; 97.11 ; 103.85$; $105.86 ; 122.65 ; 124.15 ; 125.29 ; 128.22 ; 129.02 ; 130.01$; $131.73 ; 135.97 ; 136.32 ; 137.58 ; 137.86 ; 141.70 ; 144.98$; $148.66 ; 148.93 ; 160.45 ; 171.97 ; 173.58 ; 174.20 ; 174.48$; 196.31; 211.49. UV-Vis $\left(\mathrm{CH}_{2} \mathrm{Cl}_{2}\right) \lambda_{\max } \mathrm{nm}(\varepsilon): 413(85,900)$; 507 (8,500); $538(8,000) ; 609(6,500) ; 667(35,800)$.

29. Ponomarev G.V., Solovieva M.N., Dugin N.O., Zavialova M.G., Mehtiev A.R., Misharin A.Yu., Novikov R.A., Tkachev Y.V., Popenko V.I., Timofeev V.P. Bioorg. Med. Chem. 2013, 21, 5420.

30. Mosmann T. J. Immunol. Methods 1983, 65, 55. 